\title{
Spin Physics with COMPASS
}

\author{
Jörg Pretz ${ }^{* \dagger}$ \\ CERN, Switzerland \\ E-mail: jorg.pretz@cern.ch
}

COMPASS is a fixed target experiment at CERN studying the spin structure of the nucleon via deep inelastic muon-nucleon scattering. Hadron spectroscopy and structure are investigated using hadronic beams.

The main goal of the muon program is the determination of the gluon helicity contribution, $\Delta G / G$, to the nucleon spin. Experimentally this quantity is accessible via the measurement of double spin asymmetries in two different processes: The first one is the production of hadron pairs with large transverse momentum. The second one is open charm production which provides the cleanest and most direct measurement. The first method has a higher statistical accuracy but is affected by larger systematic uncertainties due to background processes. First results from COMPASS indicate that the helicity contribution of the gluons to the nucleon spin is small.

In parallel to the $\Delta G$ measurement longitudinal inclusive and semi-inclusive asymmetries are studied as well. These allow a flavor decomposition of the helicity contribution, $\Delta \Sigma$, of the quarks to the nucleon spin. The spin physics program of COMPASS also includes measurements of single spin asymmetries on a transversely polarized target, giving access to transverse quark distributions in the nucleon.

Recent results of the muon program from the data taking periods 2002-2003 will be presented.

International Europhysics Conference on High Energy Physics

July 21st - 27th 2005

Lisboa, Portugal

*Speaker.

${ }^{\dagger}$ on behalf of the COMPASS collaboration 


\section{Introduction}

Relativistic quark models predict that the quark helicities contribute approximately $75 \%$ to the nucleon spin. Results from deep inelastic scattering (DIS) indicate a much smaller value of $\Delta \Sigma=20-30 \%$. The difference could be explained by a large helicity contribution of gluons, $\Delta G$, to the nucleon spin. This quantity is in principal accessible in next-to-leading order analysis of inclusive spin asymmetries, but this provides a rather indirect measurement of $\Delta G$ with large systematic uncertainties.

In the following direct measurements of $\Delta G$ and an improved determination of $\Delta \Sigma$ are described. Results on the transverse quark distributions $\Delta q_{T}(x)$ are discussed at the end.

The data presented were taken in the years $2002-2003$ with a $160 \mathrm{GeV}$ polarized muon beam on a longitudinally or transversely polarized ${ }^{6} \mathrm{LiD}$ target. A more detailed description of the experimental setup can be found in [1].

\section{The measurement of $\Delta G$}

Information about gluons inside the proton can be obtained in semi-inclusive deep inelastic scattering by selecting hadronic final states signaling the participation of a gluon in the underlying partonic sub-process. For example, the presence of a hadron pair with large transverse momentum (typically $p_{T}>0.7 \mathrm{GeV}$ ) with respect to the virtual photon axis tags events where the photon interacts with a gluon inside the proton via the photon-gluon fusion process. Unfortunately, other processes, like the QCD-Compton process, have the same signature leading to background contributions.

A much cleaner tag of the photon-gluon fusion process is the observation of charmed particles in the final state. Because of the small intrinsic charm contribution in the proton and the low probability to produce charm quarks in the fragmentation process, charm quarks are almost exclusively produced via the photon-gluon fusion process. Experimentally, one detects $D^{0}$ and $D^{*+}$ mesons and their anti-particles via their respective decays in $K^{-}+\pi^{+}$and $K^{-}+\pi^{+}+\pi_{\text {slow }}^{+}$.

In both methods (high $p_{T}$ and open charm) one has to measure a double spin asymmetry $A^{\text {raw }}$ (longitudinally polarized beam and target). This asymmetry is related to $\Delta G / G$ in the following way:

$$
A^{\text {raw }}=\frac{N^{\uparrow \downarrow}-N^{\uparrow \uparrow}}{N^{\uparrow \downarrow}+N^{\uparrow \uparrow}}=P_{B} P_{T} f a_{L L} \frac{\sigma_{P G F}}{\sigma_{P G F}+\sigma_{b g d}} \frac{\Delta G}{G}+A^{b g d}
$$

The meaning of the variables in eq. (2.1) and their approximate numerical values are given in tab. 1. Whereas some of the diluting factors $\left(P_{B}, P_{T}, f\right)$ are the same in both methods, some are different. $a_{L L}$, the asymmetry of the partonic photon-gluon fusion process, is for example negative for light quarks and varying from positive to negative values as a function of the photongluon center-of-mass energy for heavy quarks. Another important difference is that for the high $p_{T}$ method the background has to be estimated from Monte Carlo generators (PHYTIA for $Q^{2}<$ $1 \mathrm{GeV}^{2}$ and LEPTO for $Q^{2}>1 \mathrm{GeV}^{2}$ ), whereas in the open charm method it can be measured from the background in the invariant mass spectrum of the observed $D$ mesons. This reduces the model dependence of the result. 


\begin{tabular}{|c|c|c|}
\hline & high $p_{T}$ pairs & open-charm \\
\hline$\overline{P_{B}}$ & \multicolumn{2}{|c|}{ beam polarization $\approx-0.8$} \\
\hline$P_{T}$ & \multicolumn{2}{|c|}{ target polarization $\approx 0.5$} \\
\hline$f$ & \multicolumn{2}{|c|}{ dilution factor $\approx 0.4$ for ${ }^{6} \mathrm{LiD}$ target } \\
\hline$a_{L L}$ & \multicolumn{2}{|c|}{ asymmetry of partonic process } \\
\hline & $\approx-0.4$ & -0.5 to 0.5 \\
\hline$\frac{\sigma_{P G F}}{\sigma_{P G F}+\sigma_{h o d}}$ & \multicolumn{2}{|c|}{ fraction of photon-gluon fusion process } \\
\hline & 0.3 & $0.5\left(D^{*}\right) 0.1\left(D^{0}\right)$ \\
\hline $\begin{array}{l}\text { source of background } \\
\text { determination of bgd }\end{array}$ & $\begin{array}{l}\text { Compton, resolved photon, .. } \\
\text { LEPTO/PYTHIA MC }\end{array}$ & $\begin{array}{l}\text { combinatorial background } \\
\text { from } D^{*}\left(D^{0}\right) \text { mass spectrum }\end{array}$ \\
\hline$A^{b g d}$ & \multicolumn{2}{|c|}{ background asymmetry $\approx 0$} \\
\hline
\end{tabular}

Table 1: Explanation of the variables used in eq.(2.1).

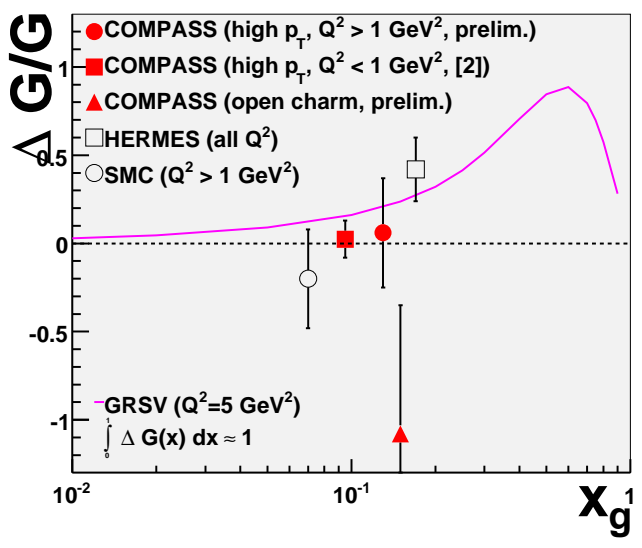

Figure 1: The result on $\Delta G / G$ as a function of the gluon momentum fraction $x_{g}$. The scale for the high $p_{t}$ points is $\approx 3 \mathrm{GeV}^{2}$ whereas it is $\approx 10 \mathrm{GeV}^{2}$ for the open charm point. For the points obtained with the high $p_{T}$ method, statistical and systematical error are shown. For the point from open charm only the dominating statistical error is shown. For the COMPASS points the $x_{g}$-range probed has an RMS of about 0.08 . The curve is a parameterization ([4]) with a first moment $\Delta G=\int_{0}^{1} \Delta G(x) \mathrm{dx}=1$.

Fig. 1 shows the results for $\Delta G / G$ obtained by COMPASS together with results from other experiments. The curve is a parameterization [4] assuming a first moment $\Delta G=\int_{0}^{1} \Delta G(x) \mathrm{dx}=1$. The results clearly favor a smaller value of $\Delta G / G$ at $x_{g} \approx 0.1$.

\section{Inclusive asymmetry $A_{1}^{d}$}

Parallel to the measurement of $\Delta G / G$, the inclusive asymmetry $A_{1}^{d}$ was determined (s. Fig. 2,[1]). At large Bjorken- $x$, the measurement agrees with previous experiments. At low $x$ COMPASS improves the precision by more than a factor 2 . In a NLO analysis of the world data on inclusive asymmetries we find $\Delta \Sigma_{\overline{M S}}=0.25 \pm 0.02$ (stat). Without the new COMPASS data the error is about $50 \%$ larger.

\section{The measurement of $\Delta_{T} q$}

The transversity distribution $\Delta_{T} q(x)$ can be accessed in semi-inclusive deep inelastic scattering on a transversely polarized target via single spin asymmetries or the measurement of the transverse polarization of produced hadrons in the final state. In general:

Asymmetry (or Polarization) $\propto \Delta_{T} q \times$ analyzing power . 


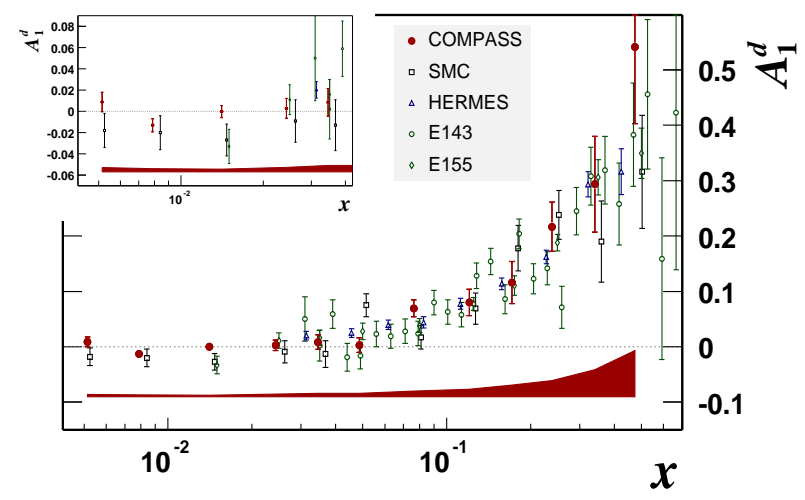

Figure 2: The inclusive asymmetry $A_{1}^{d}$ as a function of Bjorken- $x$. The measurement agrees at large $x$ with previous experiments. At low $x$ (see inlet) COMPASS improves the precision by more than a factor 2,[1].
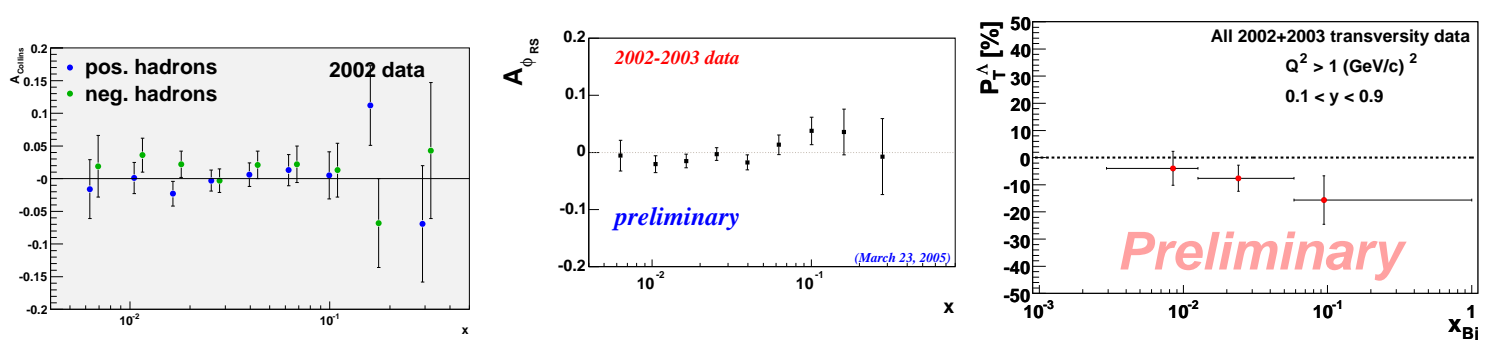

Figure 3: The Collins asymmetry for positive and negative hadrons (left,[3] ), the two hadron asymmetry (middle) and the transverse $\Lambda$-polarization (right) as a function of $x$.

COMPASS studies three different channels:

$$
\begin{aligned}
\text { Collins asymmetry: } & \propto \Sigma_{q} e_{q}^{2} \Delta_{T} q(x) \Delta_{T}^{0} D_{q}^{h}\left(z,\left|p_{T}^{h}\right|\right) \\
2 \text { hadron asymmetry: } & \propto \Sigma_{q} e_{q}^{2} \Delta_{T} q(x) H_{q}^{\angle h}\left(z, M_{h}\right) \\
\Lambda \text { - Polarization: } & \propto \Sigma_{q} e_{q}^{2} \Delta_{T} q(x) \Delta D_{q}^{\Lambda}(z)
\end{aligned}
$$

The analyzing power, i.e. the measurement of the quark's final state polarization is different for the different processes and sometimes even not well known, underlining the importance to study several independent ways to determine $\Delta_{T} q(x)$.

Fig. 3 (left) shows the Collins asymmetries, Fig. 3 (middle) the asymmetry resulting from the two hadron analysis and Fig. 3 (right) the transverse $\Lambda$ polarization. All measurements are compatible with 0 . This may be due to small values of either $\Delta_{T} q(x)$ or the corresponding analyzing powers. Another reason could be the cancellation between different quark flavors in the sum of eq. (4.2-4.4). Therefore, to make more quantitative statements on $\Delta_{T} q(x)$, measurements on a proton target are necessary.

\section{References}

[1] E. S. Ageev et al., PLB 612 (2005) 154

[2] E. S. Ageev et al., hep-ex/0511028

[3] V. Yu. Alexakhin et al., PRL 94 (2005) 202002

[4] M. Gück et al., Phys. Rev. D 63 (2001) 094005 